\title{
The Application of Virtual Reality to the Treatment of PTSD Following the WTC Attack
}

\author{
JOANN DIFEDE, ${ }^{a}$ JUDITH CUKOR,${ }^{a}$ IVY PATT,${ }^{a}$ CEZAR GIOSAN, ${ }^{a}$ \\ AND HUNTER HOFFMAN ${ }^{b}$ \\ ${ }^{a}$ Weill Medical College of Cornell University, New York, New York 10021, USA \\ ${ }^{b}$ University of Washington, Human Interface Technology Laboratory, \\ Seattle, Washington 98195, USA
}

\begin{abstract}
Recent research suggests that virtual reality (VR) enhanced exposure therapy may enhance the efficacy of treatment through increasing patient engagement in the exposure. This study evaluated the use of VR in the treatment of PTSD following the WTC attack of September 11, 2001. Individuals in a 14 session VR-enhanced treatment $(n=9)$ were compared to a waitlist (WL) control group $(n=8)$. ANOVA showed a significant interaction of time by group $(p<.01)$ with a large effect size of 1.53. The VR group showed significantly greater post-treatment decline in CAPS scores compared to the WL. Our preliminary data suggests that $V R$ is an effective tool for enhancing exposure therapy for both civilians and disaster workers who suffer from PTSD.
\end{abstract}

KEYWORDS: virtual reality; VR; posttraumatic stress disorder; September 11; exposure therapy

The efficacy of exposure therapy for the treatment of posttraumatic stress disorder (PTSD) has been established in multiple studies. ${ }^{1}$ However, the inability of some patients to emotionally engage in this type of therapy remains a challenge to its effectiveness. ${ }^{2}$ Recent research suggests that virtual reality (VR) enhanced exposure therapy may facilitate emotional engagement and, therefore, enhance the efficacy of exposure therapy. ${ }^{3}$

The goal of this pilot study is to evaluate the use of VR in the treatment of PTSD following the WTC attack of September 11, 2001. Participants were evaluated with widely used well-validated clinical interviews, including the CAPS, the SCID, a trauma history interview, and self-report measures. Those with PTSD were offered a 14-session weekly treatment that used VR technology

Address for correspondence: JoAnn Difede, Ph.D., Director, Program for Anxiety and Traumatic Stress Studies, Weill Medical College of Cornell University, 525 East 68th Street, Box 200, New York, NY 10021, Voice: 212-746-3079; fax: 212-821-0994.

e-mail: jdifede@med.cornell.edu

Ann. N.Y. Acad. Sci. 1071: 500-501 (2006). (C) 2006 New York Academy of Sciences. doi: 10.1196/annals.1364.052 
as a tool to enhance exposure therapy. The WTC virtual environment was constructed to allow for a graded hierarchical exposure to the stimuli in the world, with scenes ranging from a plane flying past the towers, to the entire reenactment of the events of $9 / 11$ with two planes crashing and the towers collapsing.

Those in the VR treatment ( $n=9)$ were compared to a waitlist control group $(n=11)$. Participants were mostly middle-aged male disaster workers. Notably, 6 of the 9 VR participants had failed to improve with imaginal exposure therapy and still suffered from PTSD with a mean baseline CAPS score of 78.0 $(S D=24.0)$ before the VR treatment was initiated. ANOVA showed a significant interaction of time by group $(P<0.05)$. Post-hoc $t$-tests showed that CAPS scores revealed a substantial decrease in the VR group $(t=3.32$, $P=0.01)$, but no significant decrease in the WL group $(t=-0.64, P=$ 0.5 ). The between group (VR verses WL) posttreatment effect size was large at 1.53. All 5 of the 8 VR subjects who had received prior treatment for PTSD showed $>25 \%$ reduction in CAPS scores post treatment, 5 of 8 subjects in the VR group no longer met criteria for PTSD, while all 8 subjects in the waitlist condition still met criteria for PTSD (chi square $=7.3, d f=1, P<0.01$ ).

Our preliminary data suggest that VR is an effective tool for enhancing exposure therapy for both civilians and disaster workers who suffer from PTSD and may be most useful for those patients who cannot engage in imaginal exposure therapy. It is notable that a standardized virtual WTC trauma environment was effective in emotionally engaging individuals with greatly varying exposure and experiences.

\section{REFERENCES}

1. Rothbaum, B.O., E.A. Meadows, P. Resick \& D.W. Foy. 2000. Cognitivebehavioral therapy. In Effective Treatments for PTSD. E.B. Foa, T.M. Keane \& M.J. Friedman, Eds: 320-325. New York. The Guilford Press.

2. JAYCOX, L.H., E.B. FOA \& A.R. MORRAL. 1998. Influence of emotional engagement and habituation on exposure therapy for PTSD. J. Consult. Clin. Psychol. 66: 86-192.

3. Rothbaum, B.O., L.F. Hodges, D. Ready, K. GraAp \& R. Alarcon. 2001. Virtual reality exposure therapy for Vietnam veterans with posttraumatic stress disorder. J. Clin. Psychiatry 62: 617-622. 